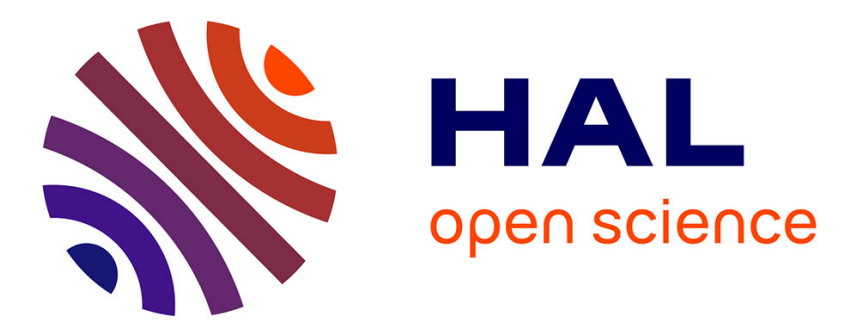

\title{
Convergence acceleration for observers by gain commutation
}

Denis Efimov, Andrey Polyakov, Arie Levant, Wilfrid Perruquetti

\section{To cite this version:}

Denis Efimov, Andrey Polyakov, Arie Levant, Wilfrid Perruquetti. Convergence acceleration for observers by gain commutation. International Journal of Control, 2018, 91 (9), pp.1-20. 10.1080/00207179.2017.1415465 . hal-01651747

\section{HAL Id: hal-01651747 \\ https://hal.inria.fr/hal-01651747}

Submitted on 29 Nov 2017

HAL is a multi-disciplinary open access archive for the deposit and dissemination of scientific research documents, whether they are published or not. The documents may come from teaching and research institutions in France or abroad, or from public or private research centers.
L'archive ouverte pluridisciplinaire HAL, est destinée au dépôt et à la diffusion de documents scientifiques de niveau recherche, publiés ou non, émanant des établissements d'enseignement et de recherche français ou étrangers, des laboratoires publics ou privés. 


\title{
Convergence acceleration for observers by gain commutation
}

\author{
D. Efimov, A. Polyakov, A. Levant, W. Perruquetti
}

\section{ARTICLE HISTORY}

Compiled November 23, 2017

\begin{abstract}
Increasing convergence rates of observers and differentiators for a class of nonlinear systems in the output canonical form (under presence of bounded matched disturbances, Lipschitz uncertainties and measurement noises) is investigated. A supervisory algorithm is designed that switches among different values of observer gains to accelerate the estimation. In the noise-free case, the presented switched-gain observer ensures global uniform time of convergence of the estimation error to the origin. In the presence of noise, the goals of overshoot reducing for the initial phase, acceleration of convergence and improvement of asymptotic precision of estimation are achieved. Efficiency of the proposed switching-gain observer is demonstrated by numerical comparison with a sliding mode and linear high-gain observers.
\end{abstract}

\section{Introduction}

Estimation of the internal state for dynamical systems is one of the central problems in the control systems theory, with posterior applications for output stabilization, monitoring or fault detection. Numerous linear estimation or observation algorithms and plenty nonlinear methods are proposed Crassidis and Junkins (2012); Khalil (1996); Luenberger (1979); Sontag (1998); Utkin (1992). The differences between them consist in the restrictions imposed on the plant model and in the provided performances for the estimation error dynamics (asymptotic precision; initial overshooting; rate and domain of convergence; robustness with respect to external disturbances, measurement noises and small delays, etc.).

In the present work a particular attention is given to improvement of convergence rates. And the idea of the proposed solution comes from the fact that if a system is homogeneous with a negative/zero/positive degree and asymptotically stable, then actually it has a finite-time/exponential/asymptotic rate of convergence (in the case of positive degree the time of convergence to a ball is globally bounded for any initial conditions, which is called fixed-time stability) Moulay and Perruquetti (2006, 2008); Polyakov (2012).

D. Efimov, A. Polyakov and W. Perruquetti are with Inria, Non-A team, Parc Scientifique de la Haute Borne, 40 avenue Halley, 59650 Villeneuve d'Ascq, France and CRIStAL (UMR-CNRS 9189), Ecole Centrale de Lille, Cit Scientifique, 59651 Villeneuve-d'Ascq, France.

D. Efimov and A. Polyakov are with Department of Control Systems and Informatics, University ITMO, 49 av. Kronverkskiy, 197101 Saint Petersburg, Russia.

A. Levant is with School of Mathematical Sciences,Tel-Aviv University, Tel-Aviv 6997801, Israel.

This work was partially supported by ANR 15 CE23 0007 (Project Finite4SoS), the Government of Russian Federation (Grant 074-U01) and the Ministry of Education and Science of Russian Federation (Project 14.Z50.31.0031). 
In applications, steady-state estimation errors and convergence rates are the main characteristics for state observers. The problem of adjustment of the convergence rate for a class of observers designed for nonlinear systems in the output canonical form is studied in this paper. It is a well-known fact that increasing the values of observer gains accelerates the speed of convergence of the estimates to the real values, however, such a strategy also leads to the robustness degradation with respect to the measurement noises and an initial huge overshooting (peaking phenomenon) Luenberger (1979). Resolving these issues, a supervisory algorithm orchestrating different values of the observer gains is given in the present work. Such a problem has been studied previously, for example, noise dependence optimization of the asymptotic precision via a single switch of the observer gains is proposed in Ahrens and Khalil (2009); Elbeheiry and Elmaraghy (2003). Continuous-time gain adaptation is investigated in many works, see for instance Andrieu, Praly, and Astolfi (2009); Boizot, Busvelle, and Gauthier (2010); Sanfelice and Praly (2011). For stabilization by state feedback several supervisory algorithms dealing with improvement of convergence rate can be found in Ananyevskii (2001, 2003); Dvir and Levant (2015a, 2015b); Efimov, Levant, Polyakov, and Perruquetti (2016, 2017). In Ananyevskii (2003), for a scalar linear system with bounded perturbation a switching rule is designed, which increases the scalar gain of linear feedback making the closed-loop system finite-time stable, an extension to planar mechanical systems is obtained in Ananyevskii (2001). In Dvir and Levant (2015a, 2015b), for sliding mode control systems Fridman (2011); Moreno and Osorio (2012); Poznyak, Fridman, and Bejarano (2004); Utkin (1992), an algorithm of online switching between parameters is proposed, which ensures a desired accelerated rate of convergence for the closed-loop system. An algorithm of parameter switching for finite-time and fixed-time convergence to the origin (or a ball) is developed in Efimov, Levant, et al. (2016); Efimov et al. (2017) for homogeneous systems with negative/zero/positive degrees. The present work is based on ideas of gain commutation and optimization presented in Ahrens and Khalil (2009); Vasiljevic and Khalil (2008) and Efimov, Levant, et al. (2016); Efimov et al. (2017), and it complements other fixed-time converging observers from Cruz-Zavala, Moreno, and Fridman (2011); Lopez-Ramirez, Efimov, Polyakov, and Perruquetti (2016); Ríos and Teel (2016).

The outline of this paper is as follows. Notation and preliminary results are introduced in sections 2 and 3, respectively. The precise problem statement and some auxiliary results are given in Section 4. The proposed supervisory algorithm of switching among different values of the observer gain, which ensures a required acceleration, is presented in Section 5. Demonstration of performances and comparison of the proposed supervisory algorithm with a sliding-mode and linear differentiators are discussed in Section 6. Concluding remarks appear in Section 7.

\section{Notation}

Through the paper the following notation is used:

- $\mathbb{N}$ is the set of nonnegative natural numbers and $\mathbb{R}_{+}=\{x \in \mathbb{R}: x \geq 0\}$, where $\mathbb{R}$ is the set of real number.

- $|\cdot|$ denotes the absolute value in $\mathbb{R},\|\cdot\|$ denotes the Euclidean norm in $\mathbb{R}^{n}$, $\|x\|_{\mathcal{A}}=\inf _{\xi \in \mathcal{A}}\|x-\xi\|$ is the distance from a point $x \in \mathbb{R}^{n}$ to a set $\mathcal{A} \subset \mathbb{R}^{n}$.

- For a (Lebesgue) measurable function $d: \mathbb{R}_{+} \rightarrow \mathbb{R}^{m}$ define the norm $\|d\|_{\left[t_{0}, t_{1}\right)}=$ $\left.\operatorname{ess}_{\sup } \operatorname{sit}_{0}, t_{1}\right)\|d(t)\|$, then $\|d\|_{\infty}=\|d\|_{[0,+\infty)}$ and the set of $d(t)$ with the pro- 
perty $\|d\|_{\infty}<+\infty$ we further denote as $\mathcal{L}_{\infty}^{m}$ (the set of essentially bounded measurable functions from $\mathbb{R}_{+}$to $\left.\mathbb{R}^{m}\right) ; \mathcal{L}_{D}^{m}=\left\{d \in \mathcal{L}_{\infty}^{m}:\|d\|_{\infty} \leq D\right\}$ for any $D>0$.

- For a symmetric matrix $A \in \mathbb{R}^{n \times n}$ denote $\lambda_{\min }(A)$ and $\lambda_{\max }(A)$ the minimum and the maximum eigenvalues of $A$, respectively.

- $I_{n}$ is $n \times n$ identity matrix.

\section{Preliminaries}

Consider the following nonlinear system:

$$
\dot{x}(t)=f(x(t), d(t)), t \geq 0,
$$

where $x(t) \in \mathbb{R}^{n}$ is the state, $d(t) \in \mathbb{R}^{m}$ is the input, $d \in \mathcal{L}_{\infty}^{m} ; f: \mathbb{R}^{n+m} \rightarrow \mathbb{R}^{n}$ ensures forward existence of the system solutions (understood in the Filippov sense Filippov (1988)) at least locally, $f(0,0)=0$. For an initial condition $x_{0} \in \mathbb{R}^{n}$ and input $d \in \mathcal{L}_{\infty}^{m}$ define the corresponding solution by $X\left(t, x_{0}, d\right)$ for any $t \geq 0$ for which the solution exists. A set $\mathcal{A} \subset \mathbb{R}^{n}$ is called uniformly forward invariant for (1) if $x_{0} \in \mathcal{A}$ implies that $X\left(t, x_{0}, d\right) \in \mathcal{A}$ for all $t \geq 0$ and all $d \in \mathcal{L}_{D}^{m}$ for given $D>0$.

Following Khalil (1996); Lin, Sontag, and Wang (1996); Polyakov (2012); Roxin (1966), let $\Omega$ be an open neighborhood of non-empty, compact and uniformly forward invariant set $\mathcal{A} \subset \mathbb{R}^{n}$ of (1) with some $D>0$.

Definition 3.1. At the set $\mathcal{A}$ the system (1) for $d \in \mathcal{L}_{D}^{m}$ is said to be

(a) uniformly Lyapunov stable if for any $x_{0} \in \Omega$ and $d \in \mathcal{L}_{D}^{m}$ the solutions $X\left(t, x_{0}, d\right)$ are defined for all $t \geq 0$, and for any $\epsilon>0$ there is $\delta>0$ such that for any $x_{0} \in \Omega$, if $\left\|x_{0}\right\|_{\mathcal{A}} \leq \delta$ then $\left\|X\left(t, x_{0}, d\right)\right\|_{\mathcal{A}} \leq \epsilon$ for all $t \geq 0$;

(b) uniformly asymptotically stable if it is uniformly Lyapunov stable and for any $\kappa>0$ and $\epsilon>0$ there exists $T(\kappa, \epsilon) \geq 0$ such that for any $x_{0} \in \Omega$ and $d \in \mathcal{L}_{D}^{m}$, if $\left\|x_{0}\right\|_{\mathcal{A}} \leq \kappa$ then $\left\|X\left(t, x_{0}, d\right)\right\|_{\mathcal{A}} \leq \epsilon$ for all $t \geq T(\kappa, \epsilon)$;

(c) uniformly finite-time stable if it is uniformly Lyapunov stable and uniformly finite-time converging from $\Omega$ : for any $x_{0} \in \Omega$ and all $d \in \mathcal{L}_{D}^{m}$ there exists $0 \leq T<+\infty$ such that $X\left(t, x_{0}, d\right) \in \mathcal{A}$ for all $t \geq T$. The function $T_{\mathcal{A}}\left(x_{0}\right)=\inf \{T \geq 0: \forall t \geq$ $\left.T, \forall d \in \mathcal{L}_{D}^{m} X\left(t, x_{0}, d\right) \in \mathcal{A}\right\}$ is called the uniform settling time of the system (1);

(d) uniformly fixed-time stable if it is uniformly finite-time stable and $\sup _{x_{0} \in \Omega} T_{\mathcal{A}}\left(x_{0}\right)<+\infty$.

The set $\Omega$ is called a domain of stability/attraction.

If $\Omega=\mathbb{R}^{n}$, then the corresponding properties are called global uniform Lyapunov/asymptotic/finite-time/fixed-time stability of (1) for $d \in \mathcal{L}_{D}^{m}$ at $\mathcal{A}$.

\section{Problem introduction}

In this section the system of interest is presented with a basic observer, next some their properties used in the sequel are discussed, and finally the problem statement is given. 


\subsection{Basic system and its observer}

Consider a nonlinear system in a canonical form:

$$
\begin{aligned}
\dot{x}(t)= & A x(t)+\varphi(u(t), y(t)) \\
& +b[g(x(t))+d(t)], t \geq 0, \\
y(t)= & c^{T} x(t)+v(t),
\end{aligned}
$$

where $x(t) \in \mathbb{R}^{n}$ is the state vector with $n>1, y(t) \in \mathbb{R}$ is the output available for measurements with the noise $v(t) \in \mathbb{R}, v \in \mathcal{L}_{V}$ for some $V>0 ; u(t) \in \mathbb{R}^{m}$ is control, $u \in \mathcal{L}_{\infty}^{m} ; d(t) \in \mathbb{R}$ is the exogenous disturbance, $d \in \mathcal{L}_{D}$ for some $D>0$; the matrices

$$
A=\left[\begin{array}{cccc}
0 & 1 & 0 \ldots 0 & 0 \\
0 & 0 & 1 \ldots 0 & 0 \\
\vdots & \vdots & \ddots & \vdots \\
0 & 0 & 0 \ldots 0 & 1 \\
0 & 0 & 0 \ldots 0 & 0
\end{array}\right], b=\left[\begin{array}{c}
0 \\
\vdots \\
0 \\
1
\end{array}\right], c=\left[\begin{array}{c}
1 \\
0 \\
\vdots \\
0
\end{array}\right]
$$

are in the canonical form; the function $\varphi: \mathbb{R}^{m+1} \rightarrow \mathbb{R}^{n}$ ensures existence and uniqueness of solutions of the system (2), the function $g: \mathbb{R}^{n} \rightarrow \mathbb{R}$ is globally Lipschitz, then there exists $\gamma>0$ such that for all $x^{\prime}, x \in \mathbb{R}^{n}$ :

$$
\left\|g(x)-g\left(x^{\prime}\right)\right\| \leq \gamma\left\|x-x^{\prime}\right\| .
$$

Following Ahrens and Khalil (2009); Khalil (1996); Sanfelice and Praly (2011) the simplest observer for (2) takes the form:

$$
\begin{aligned}
\dot{\hat{x}}(t)= & A \hat{x}(t)+\varphi(u(t), y(t))+b g(\hat{x}(t)) \\
& +l\left(y(t)-c^{T} \hat{x}(t)\right),
\end{aligned}
$$

where $\hat{x}(t) \in \mathbb{R}^{n}$ is the estimate of $x(t)$ and $l \in \mathbb{R}^{n}$ is the observer gain to be designed.

Assumption 4.1. For given $\gamma>0, \kappa>0, \rho_{d}>0$ and $\rho_{v}>0$ there exist $P=P^{T} \in$ $\mathbb{R}^{n \times n}$ and $w \in \mathbb{R}^{n}$ such that

$$
\begin{gathered}
P>0,\left[\begin{array}{cccc}
\tilde{S} & P b & -w & -P b \\
b^{T} P & -\rho_{d}^{2} & 0 & 0 \\
-w^{T} & 0 & -\rho_{v}^{2} & 0 \\
-b^{T} P & 0 & 0 & -\gamma^{-2}
\end{array}\right] \leq 0, \\
\tilde{S}=A^{T} P+P A-c w^{T}-w c^{T}+I_{n}+\kappa P .
\end{gathered}
$$

In this assumption $\kappa, \rho_{d}$ and $\rho_{v}$ are design parameters, which meaning will be explained later.

Remark 1. For $\gamma=0$, i.e. if there is no uncertainty $g(\cdot)$, then the above linear matrix 
inequalities (LMIs) take the form:

$$
\begin{gathered}
P>0, \quad\left[\begin{array}{ccc}
\tilde{S} & P b & -w \\
b^{T} P & -\rho_{d}^{2} & 0 \\
-w^{T} & 0 & -\rho_{v}^{2}
\end{array}\right] \leq 0, \\
\tilde{S}=A^{T} P+P A-c w^{T}-w c^{T}+\kappa P .
\end{gathered}
$$

Lemma 4.2. Let Assumption 4.1 be satisfied, then in (2), (3) with $l=P^{-1} w$ for all $t \geq 0$ :

$$
\begin{aligned}
\|x(t)-\hat{x}(t)\| \leq & \sqrt{\frac{\lambda_{\max }(P)}{\lambda_{\min }(P)}} e^{-0.5 \kappa t}\|x(0)-\hat{x}(0)\| \\
& +\sqrt{\frac{1}{\kappa \lambda_{\min }(P)}}\left(\rho_{d} D+\rho_{v} V\right) .
\end{aligned}
$$

Proof. The estimation error $e(t)=x(t)-\hat{x}(t)$ has the following dynamics:

$$
\dot{e}(t)=\left(A-l c^{T}\right) e(t)+b[g(x(t))-g(\hat{x}(t))+d(t)]-l v(t) .
$$

Consider a Lyapunov function candidate $W(e)=e^{T} P e$, where $P=P^{T}>0$ is a matrix introduced in Assumption 4.1, then

$$
\begin{aligned}
\dot{W}= & e^{T}\left\{\left(A-l c^{T}\right)^{T} P+P\left(A-l c^{T}\right)\right\} e \\
& +2 e^{T} P b[g(x)-g(\hat{x})+d]-2 e^{T} P l v .
\end{aligned}
$$

Note that

$$
\begin{gathered}
2 e^{T} P b[g(x)-g(\hat{x})] \leq \gamma^{2} e^{T} P b b^{T} P e+\gamma^{-2}\|g(x)-g(\hat{x})\|^{2} \\
\leq \gamma^{2} e^{T} P b b^{T} P e+e^{T} e=e^{T}\left[\gamma^{2} P b b^{T} P+I_{n}\right] e
\end{gathered}
$$

then

$$
\begin{aligned}
\dot{W} \leq & e^{T}\left\{\left(A-l c^{T}\right)^{T} P+P\left(A-l c^{T}\right)+\gamma^{2} P b b^{T} P\right. \\
& \left.+I_{n}\right\} e+2 e^{T} P b d-2 e^{T} P l v \\
= & {\left[\begin{array}{c}
e \\
d \\
v
\end{array}\right]^{T} Q\left[\begin{array}{l}
e \\
d \\
v
\end{array}\right]-\kappa W+\rho_{d}^{2} d^{2}+\rho_{v}^{2} v^{2}, }
\end{aligned}
$$

where $\kappa>0, \rho_{d}>0$ and $\rho_{v}>0$ are design parameters, and

$$
\begin{gathered}
Q=\left[\begin{array}{ccc}
S & P b & -P l \\
b^{T} P & -\rho_{d}^{2} & 0 \\
-l^{T} P & 0 & -\rho_{v}^{2}
\end{array}\right], \\
S=\left(A-l c^{T}\right)^{T} P+P\left(A-l c^{T}\right)+\gamma^{2} P b b^{T} P+I_{n}+\kappa P .
\end{gathered}
$$


If $Q \leq 0$, then we get the inequality

$$
\dot{W} \leq-\kappa W+\rho_{d}^{2} d^{2}+\rho_{v}^{2} v^{2},
$$

which implies that the estimation error dynamics is input-to-state stable with respect to external disturbance $d$ and measurement noise $v$. Introducing variable $w=P l$ and applying Schur complement, it is straightforward to verify that fulfillment of LMIs given in Assumption 4.1 guarantees the condition $Q \leq 0$ with $l=P^{-1} w$. From the properties of $W$ we obtain:

$$
\begin{gathered}
\lambda_{\min }(P)\|e\|^{2} \leq W(e) \leq \lambda_{\max }(P)\|e\|^{2}, \\
W(t) \leq e^{-\kappa t} W(0)+\kappa^{-1}\left(\rho_{d}^{2} D^{2}+\rho_{v}^{2} V^{2}\right),
\end{gathered}
$$

where $W(t)=W(e(t))$, from which the required estimate follows.

\subsection{The problem statement}

As it has been established in Lemma 4.2, the observer (3) ensures a robust estimation of the state of (2) with an exponential rate of convergence (if LMIs from Assumption 4.1 are satisfied). The problem further studied in this work is to ensure the uniform fixed-time estimation of (2), with convergence of estimation error to a ball proportional to $D$ and $V$, by switching among different sets of coefficients in the gain $l$, for $d \in \mathcal{L}_{D}$ and $v \in \mathcal{L}_{V}$ with given $D>0$ and $V>0$. Such a problem in stabilization context has been already considered in Ananyevskii (2001, 2003) (finite-time case), in Dvir and Levant (2015a, 2015b) for the sliding mode feedback, and in Efimov, Levant, et al. (2016); Efimov et al. (2017) the general case of homogeneous systems is analyzed. A high-gain observer similar to (3) with a switched gain has been proposed in Ahrens and Khalil (2009), but there the switching is performed just once in order to minimize influence of noise on asymptotic behavior. The problem of multiple commutation of gains, with accelerating the convergence and making it uniform over $\mathbb{R}^{n}$, is first time considered in the present work.

Following Ahrens and Khalil (2009); Efimov, Levant, et al. (2016), instead of (3) we will consider for all $i \in \mathbb{N}$ the observer:

$$
\begin{aligned}
\dot{\hat{x}}(t)= & A \hat{x}(t)+\varphi(u(t), y(t))+b g(\hat{x}(t)) \\
& +\mu_{i} M_{i}^{-1} l\left(y(t)-c^{T} M_{i} \hat{x}(t)\right), t \in\left[t_{i}, t_{i+1}\right),
\end{aligned}
$$

where $\hat{x}(t) \in \mathbb{R}^{n}$ has the same meaning as previously, $l$ is comes from Assumption 4.1 as before; $M_{i}=\operatorname{diag}\left\{\mu_{i}^{1-k}\right\}_{k=1}^{n}$ and scalars $\mu_{i} \geq 1$ form a sequence of parameters, which stay constant on the interval $\left[t_{i}, t_{i+1}\right)$ and change their values at instants $t_{i}$ $\left(t_{0}=0\right)$ (the system solutions are understood in the sense of Goebel, Sanfelice, and Teel (2009)).

It is required to determine the instants $t_{i}, i \in \mathbb{N}$ and the discrete-time update law for $\mu_{i}$ such that for (4) the estimation error variable $e(t)=x(t)-\hat{x}(t)$ becomes uniformly fixed-time stable with respect to the origin or a ball.

Remark 2. Note that in the case of fixed-time convergence the switching stops in a finite time $t_{*}=\sup _{i \in \mathbb{N}} t_{i}$, and the solutions of (4) have to be extended for $t \in\left[t_{*},+\infty\right)$. 
To this end, for the case $D=V=0$ the system (4) will be understood as follows:

$$
\dot{\hat{x}}(t)=\left\{\begin{array}{ll}
A \hat{x}(t)+\varphi(u(t), y(t))+b g(\hat{x}(t)) & t<t_{*} \\
+\mu_{i} M_{i}^{-1} l\left(y(t)-c^{T} M_{i} \hat{x}(t)\right), t \in\left[t_{i}, t_{i+1}\right), & \\
A \hat{x}(t)+\varphi(u(t), y(t))+b g(\hat{x}(t)) & t>t_{*}
\end{array} .\right.
$$

\section{Design of switched-gain observer}

Let us introduce an auxiliary dynamical system for $t \in\left[t_{i}, t_{i+1}\right)$ :

$$
\begin{aligned}
\dot{z}(t)= & \mu_{i}\left\{\left(A-l c^{T}\right) z(t)-l v(t)+\mu_{i}^{-n} b[g(x(t))\right. \\
& \left.\left.-g\left(x(t)-M_{i}^{-1} z(t)\right)+d(t)\right]\right\},
\end{aligned}
$$

where $\mu_{i}$ is the same as in (4), and after update of $\mu_{i}$ to $\mu_{i+1}$ at the instant of time $t_{i+1}$ we have a state resetting for $z(t)$ :

$$
z\left(t_{i+1}\right)=M_{i+1} M_{i}^{-1} z\left(t_{i+1}^{-}\right),
$$

where $z\left(t_{i+1}^{-}\right)$denotes the left limit of $z(t)$ as $t$ is approaching $t_{i+1}$ from the left. As we can conclude, (6), (7) is a hybrid system, which has to be augmented by rules for assignment of switching instants $t_{i}$ and for update of $\mu_{i}$, for all $i \in \mathbb{N}$.

It is straightforward to verify that $e(t)=M_{i}^{-1} z(t)$ for $t \in\left[t_{i}, t_{i+1}\right)$ is the corresponding solution of the estimation error dynamics of (4):

$$
\begin{aligned}
\dot{e}(t)= & \left(A-\mu_{i} M_{i}^{-1} l c^{T} M_{i}\right) e(t)+b[g(x(t)) \\
& -g(\hat{x}(t))+d(t)]-\mu_{i} M_{i}^{-1} l v(t) .
\end{aligned}
$$

Therefore, in order to design a supervisory algorithms for selection of $t_{i}$ and $\mu_{i}$ we will consider below the hybrid system (6), (7).

Lemma 5.1. Let Assumption 4.1 be satisfied, then in (6) with $l=P^{-1} w$ for all $t \in\left[t_{i}, t_{i+1}\right):$

$$
\begin{aligned}
\|z(t)\| \leq & e^{-0.5 \kappa \mu_{i}\left(t-t_{i}\right)} \sqrt{\frac{\lambda_{\max }(P)}{\lambda_{\min }(P)}}\left\|z\left(t_{i}\right)\right\| \\
& +\sqrt{\frac{1-e^{-\kappa \mu_{i}\left(t-t_{i}\right)}}{\kappa \lambda_{\min }(P)}}\left(\rho_{d} \mu_{i}^{-n} D+\rho_{v} V\right) .
\end{aligned}
$$

Proof. Consider $W(z)=z^{T} P z$, then

$$
\begin{aligned}
\dot{W}= & \mu_{i}\left\{z^{T}\left[\left(A-l c^{T}\right)^{T} P+P\left(A-l c^{T}\right)\right] z\right. \\
& +2 \mu_{i}^{-n} z^{T} P b\left[g(x)-g\left(x-M_{i}^{-1} z\right)\right. \\
& \left.+d]-2 z^{T} P l v\right\}
\end{aligned}
$$


and repeating the same steps as in the proof of Lemma 4.2 we obtain

$$
\begin{gathered}
2 \mu_{i}^{-n} z^{T} P b\left[g(x)-g\left(x-M_{i}^{-1} z\right)\right] \leq \gamma^{2} z^{T} P b b^{T} P z \\
+\gamma^{-2} \mu_{i}^{-2 n}\left\|g(x)-g\left(x-M_{i}^{-1} z\right)\right\|^{2} \\
\leq \gamma^{2} z^{T} P b b^{T} P z+\mu_{i}^{-2 n} z^{T} M_{i}^{-2} z \\
\leq z^{T}\left[\gamma^{2} P b b^{T} P+\mu_{i}^{-2} I_{n}\right] z
\end{gathered}
$$

then

$$
\begin{aligned}
\dot{W} \leq & \mu_{i}\left\{z ^ { T } \left[\left(A-l c^{T}\right)^{T} P+P\left(A-l c^{T}\right)+\gamma^{2} P b b^{T} P\right.\right. \\
& \left.\left.+\mu_{i}^{-2} I_{n}\right] z+2 \mu_{i}^{-n} z^{T} P b d-2 z^{T} P l v\right\} \\
= & \mu_{i}\left[\begin{array}{c}
e \\
\mu_{i}^{-n} d \\
v
\end{array}\right]^{T} Q_{\mu_{i}}\left[\begin{array}{c}
e \\
\mu_{i}^{-n} d \\
v
\end{array}\right]-\kappa \mu_{i} W \\
& +\rho_{d}^{2} \mu_{i}^{-2 n+1} d^{2}+\rho_{v}^{2} \mu_{i} v^{2},
\end{aligned}
$$

where

$$
\begin{gathered}
Q_{\mu_{i}}=\left[\begin{array}{ccc}
S_{\mu_{i}} & P b & -P l \\
b^{T} P & -\rho_{d}^{2} & 0 \\
-l^{T} P & 0 & -\rho_{v}^{2}
\end{array}\right], \\
S_{\mu_{i}}=\left(A-l c^{T}\right)^{T} P+P\left(A-l c^{T}\right)+\gamma^{2} P b b^{T} P \\
+\mu_{i}^{-2} I_{n}+\kappa P .
\end{gathered}
$$

It is straightforward to verify that for all $\mu_{i} \geq 1$ the property $Q_{\mu_{i}} \leq 0$ follows the condition $Q \leq 0$ provided by Assumption 4.1 for $l=P^{-1} w$, therefore

$$
\dot{W} \leq-\kappa \mu_{i} W+\rho_{d}^{2} \mu_{i}^{-2 n+1} d^{2}+\rho_{v}^{2} \mu_{i} v^{2}
$$

and for all $t \in\left[t_{i}, t_{i+1}\right)$

$$
\begin{aligned}
W(t) \leq & e^{-\kappa \mu_{i}\left(t-t_{i}\right)} W\left(t_{i}\right) \\
& +\frac{1-e^{-\kappa \mu_{i}\left(t-t_{i}\right)}}{\kappa}\left(\rho_{d}^{2} \mu_{i}^{-2 n} D^{2}+\rho_{v}^{2} V^{2}\right),
\end{aligned}
$$

where $W(t)=W(z(t))$, from which the required estimate follows.

Thus, for the system (6) augmenting value of $\mu_{i} \geq 1$ leads to increase of the convergence speed and to decrease of the gain with respect to the disturbance $d$ (the system becomes uniform in $d$ for $\left.\lim _{i \rightarrow+\infty} \mu_{i}=+\infty\right)$. 
Corollary 5.2. Let Assumption 4.1 be satisfied, then for all $t \in\left[t_{i}, t_{i+1}\right)$ :

$$
\begin{aligned}
\|x(t)-\hat{x}(t)\| \leq & \mu_{i}^{n-1} e^{-0.5 \kappa \mu_{i}\left(t-t_{i}\right)} \sqrt{\frac{\lambda_{\max }(P)}{\lambda_{\min }(P)}}\left\|x\left(t_{i}\right)-\hat{x}\left(t_{i}\right)\right\| \\
& +\sqrt{\frac{1-e^{-\kappa \mu_{i}\left(t-t_{i}\right)}}{\kappa \lambda_{\min }(P)}}\left(\rho_{d} \mu_{i}^{-1} D+\mu_{i}^{n-1} \rho_{v} V\right), \\
\mid c^{T}[x(t)-\hat{x}(t)] \leq \leq & e^{-0.5 \kappa \mu_{i}\left(t-t_{i}\right)} \sqrt{\frac{\lambda_{\max }(P)}{\lambda_{\min }(P)}}\left\|x\left(t_{i}\right)-\hat{x}\left(t_{i}\right)\right\| \\
& +\sqrt{\frac{1-e^{-\kappa \mu_{i}\left(t-t_{i}\right)}}{\kappa \lambda_{\min }(P)}}\left(\rho_{d} \mu_{i}^{-n} D+\rho_{v} V\right) .
\end{aligned}
$$

Proof. Since $e=M_{i}^{-1} z$ we have:

$$
\mu_{i}^{1-n}\|e\| \leq\|z\| \leq\|e\|,
$$

which with the estimate of Lemma 5.1 gives the first estimate. The second one follows the observation that

$$
\|z\| \geq\left|z_{1}\right|=\left|c^{T} e\right|
$$

i.e., the output is not transformed by $M_{i}$.

Thus, for the system (2), (4) augmenting value of $\mu_{i} \geq 1$ leads to increase of the convergence speed and overshooting, and to decrease of the gain with respect to the disturbance $d$, however, at the price that the gain with respect to the measurement noise $v$ grows drastically. An important observation is that the measured part of the estimation error $c^{T}[x(t)-\hat{x}(t)]$ is free from these shortages (noise gain and overshooting growth), while inheriting acceleration of the convergence rate and decrease of the disturbance gain.

\subsection{Supervisory algorithm for the noise-free case}

First, assume that $V=0$, i.e. there is no measurement noise $v(t)$ in (2). Then, according to Corollary 5.2, augmentation of $\mu_{i}$ will lead to uniform in $d$ estimation of the state of the system (2) by the observer (4). Let us design an algorithm for commutation of $\mu_{i}$ guaranteeing the global fixed-time convergence of the estimation error to the origin in $(2),(4)$.

For this purpose, the following algorithm is proposed in this work:

$$
\begin{gathered}
t_{i+1}=t_{i}+T_{i}, t_{0}=0 \\
T_{i}=-\frac{2}{\kappa \mu_{i}} \ln \left(\sqrt{\frac{\lambda_{\min }(P)}{\lambda_{\max }(P)}} \frac{\mu_{i}^{1-n}}{q}\right)
\end{gathered}
$$

for all $i \in \mathbb{N}$, where $q>1$ is a tuning parameter, and

$$
\mu_{i}=q^{\alpha i},
$$


where $\alpha>0$ is another tuning parameters. Note that for (9),

$$
\begin{aligned}
\left\|M_{i+1} M_{i}^{-1}\right\| & =\left\|\operatorname{diag}\left\{\frac{\mu_{i+1}^{1-k}}{\mu_{i}^{1-k}}\right\}_{k=1}^{n}\right\| \\
& =\left\|\operatorname{diag}\left\{q^{\alpha(1-k)}\right\}_{k=1}^{n}\right\| \leq 1
\end{aligned}
$$

and in variable $z(t)$ the state jumps at instants $t_{i}$ are not stretching, thus all properties are predefined by the continuous-time dynamics, which, as we already recognized above, is just accelerated by $\mu_{i}$.

Let us prove that for the supervisory algorithm (8), (9) the estimation error of (2), (4) converges in a fixed time to the origin.

Theorem 5.3. For $V=0$ consider the system (2), the observer (4) and the supervisory algorithm (8), (9) with $q>1$ and $\alpha \in\left(0, \frac{1}{n-1}\right]$. Then for any $D>0$ the estimation error is globally fixed-time convergent for all $d \in \mathcal{L}_{D}$. If $D=V=0$, then the solutions are extended as in Remark 2 and the estimation error dynamics is globally fixed-time stable at the origin. In addition, the time of convergence is less than

$$
\bar{T}_{0}=\frac{2}{\kappa} \frac{q^{\alpha}}{q^{\alpha}-1} \ln \left(\sqrt{\frac{\lambda_{\max }(P)}{\lambda_{\min }(P)}} q^{1+\frac{\alpha(n-1)}{q^{\alpha}-1}}\right) .
$$

Proof. Consider the estimate from Corollary 5.2 for this case for all $t \in\left[t_{i}, t_{i+1}\right)$ :

$$
\begin{aligned}
\|e(t)\| \leq & \mu_{i}^{n-1} e^{-0.5 \kappa \mu_{i}\left(t-t_{i}\right)} \sqrt{\frac{\lambda_{\max }(P)}{\lambda_{\min }(P)}}\left\|e\left(t_{i}\right)\right\| \\
& +\sqrt{\frac{1-e^{-\kappa \mu_{i}\left(t-t_{i}\right)}}{\kappa \lambda_{\min }(P)}} \frac{\rho_{d}}{\mu_{i}} D,
\end{aligned}
$$

where $e(t)=x(t)-\hat{x}(t)$ is the estimation error. If $t_{i+1}=t_{i}+T_{i}$ in accordance with (8) and $\mu_{i}$ is assigned by (9), then for any $i \in \mathbb{N}$

$$
\begin{aligned}
\left\|e\left(t_{i+1}\right)\right\| & \leq q^{-1}\left\|e\left(t_{i}\right)\right\|+\sqrt{\frac{1-\frac{\lambda_{\min }(P)}{\lambda_{\max }(P)}\left(\frac{\mu_{i}^{1-n}}{\kappa \lambda_{\min }}\right)^{2}}{\mu^{2}}} \frac{\rho_{d}}{\mu_{i}} D \\
& =q^{-1}\left\|e\left(t_{i}\right)\right\|+\sqrt{\frac{1-\frac{\lambda_{\min }(P)}{\lambda_{\max }(P)} q^{(i \alpha(1-n)-1) 2}}{\kappa \lambda_{\min }(P)}} \frac{\rho_{d}}{q^{i \alpha}} D \\
& \leq q^{-1}\left\|e\left(t_{i}\right)\right\|+\sqrt{\frac{1-\frac{\lambda_{\min }(P)}{\lambda_{\max }(P)} q^{-(i+1) 2}}{\kappa \lambda_{\min }(P)}} \frac{\rho_{d}}{q^{i \alpha}} D
\end{aligned}
$$

Repeating this operation $k>0$ times we obtain:

$$
\left\|e\left(t_{i+k}\right)\right\| \leq q^{-k}\left\|e\left(t_{i}\right)\right\|+\sqrt{\frac{1}{\kappa \lambda_{\min }(P)}} \rho_{d} D q^{-i \alpha} \sum_{s=0}^{k-1} q^{-s-(k-1-s) \alpha}
$$

for any $i \in \mathbb{N}$. Then taking $i+k \rightarrow+\infty$ and considering asymptotic behavior of 
$\left\|e\left(t_{i+k}\right)\right\|$ we get:

$$
\lim _{s \rightarrow+\infty}\left\|e\left(t_{s}\right)\right\|=0
$$

since $\lim _{k \rightarrow+\infty} \sum_{s=0}^{k-1} q^{-s-(k-1-s) \alpha} \leq \sum_{s=0}^{+\infty} q^{-s} \leq \frac{q}{q-1}$ for any $q>1$, and the system is converging to the origin uniformly in $d \in \mathcal{L}_{D}$. To evaluate the time of convergence we have to consider

$$
\begin{aligned}
\sum_{i=0}^{+\infty} T_{i}= & -\sum_{i=0}^{+\infty} \frac{2}{\kappa \mu_{i}} \ln \left(\sqrt{\frac{\lambda_{\min }(P)}{\lambda_{\max }(P)}} \frac{\mu_{i}^{1-n}}{q}\right) \\
= & -\sum_{i=0}^{+\infty} \frac{2}{\kappa q^{i \alpha}} \ln \left(\sqrt{\frac{\lambda_{\min }(P)}{\lambda_{\max }(P)}} q^{i \alpha(1-n)-1}\right) \\
= & -\sum_{i=0}^{+\infty} \frac{2}{\kappa q^{i \alpha}}\left[\ln \left(\sqrt{\frac{\lambda_{\min }(P)}{\lambda_{\max }(P)}}\right)-\ln (q)\right] \\
& -\sum_{i=0}^{+\infty} \frac{2}{\kappa q^{i \alpha}} \ln (q) i \alpha(1-n) \\
\leq & \frac{2}{\kappa} \frac{q^{\alpha}}{q^{\alpha}-1}\left[\ln \left(q^{1+\frac{\alpha(n-1)}{q^{\alpha}-1}}\right)-\ln \sqrt{\frac{\lambda_{\min }(P)}{\lambda_{\max }(P)}}\right],
\end{aligned}
$$

where the last step has been derived using the properties $\sum_{s=0}^{+\infty} q^{-\alpha s}=\frac{q^{\alpha}}{q^{\alpha}-1}$ and $\sum_{s=0}^{+\infty} s q^{-\alpha s}=\frac{q^{\alpha}}{\left(q^{\alpha}-1\right)^{2}}$ for $q>1$, and the expression in the square brackets is positive. Therefore, $\sum_{i=0}^{+\infty} T_{i} \leq \bar{T}_{0}$ and the error converges to the origin in a fixed time.

Consider now the case with $D=0$, and let us show also stability of the estimation error in (2), (4) (in this case according to Remark 2 the equation (4) is understood as (5)). From the estimate of Corollary 5.2 for this case:

$$
\begin{aligned}
\|e(t)\| & \leq \mu_{i}^{n-1} e^{-0.5 \kappa \mu_{i}\left(t-t_{i}\right)} \sqrt{\frac{\lambda_{\max }(P)}{\lambda_{\min }(P)}}\left\|e\left(t_{i}\right)\right\| \\
& \leq q^{i \alpha(n-1)} e^{-0.5 \kappa \mu_{i}\left(t-t_{i}\right)} \sqrt{\frac{\lambda_{\max }(P)}{\lambda_{\min }(P)}}\left\|e\left(t_{i}\right)\right\| \\
& \leq q^{i} \sqrt{\frac{\lambda_{\max }(P)}{\lambda_{\min }(P)}}\left\|e\left(t_{i}\right)\right\|
\end{aligned}
$$


for all $t \in\left[t_{i}, t_{i+1}\right)$ with $\alpha \in\left(0, \frac{1}{n-1}\right]$. Since $\left\|e\left(t_{i+1}\right)\right\| \leq q^{-1}\left\|e\left(t_{i}\right)\right\|$, then

$$
\begin{aligned}
\|e(t)\| & \leq \mu_{i+1}^{n-1} e^{-0.5 \kappa \mu_{i+1}\left(t-t_{i+1}\right)} \sqrt{\frac{\lambda_{\max }(P)}{\lambda_{\min }(P)}}\left\|e\left(t_{i+1}\right)\right\| \\
& \leq q^{(i+1) \alpha(n-1)} \sqrt{\frac{\lambda_{\max }(P)}{\lambda_{\min }(P)}}\left\|e\left(t_{i+1}\right)\right\| \\
& \leq q^{(i+1) \alpha(n-1)-1} \sqrt{\frac{\lambda_{\max }(P)}{\lambda_{\min }(P)}}\left\|e\left(t_{i}\right)\right\| \\
& \leq q^{i} \sqrt{\frac{\lambda_{\max }(P)}{\lambda_{\min }(P)}}\left\|e\left(t_{i}\right)\right\|
\end{aligned}
$$

for all $t \in\left[t_{i+1}, t_{i+2}\right)$, and the upper bound on $\|e(t)\|$ is not growing and it stays the same on both intervals $\left[t_{i}, t_{i+1}\right)$ and $\left[t_{i+1}, t_{i+2}\right)$. Recursively repeating these computations from $i=0$ and taking into account that after the convergence $e(t)=0$ for all $t \geq t_{*}=\sum_{i=0}^{+\infty} T_{i}$, we obtain that

$$
\|e(t)\| \leq \sqrt{\frac{\lambda_{\max }(P)}{\lambda_{\min }(P)}}\|e(0)\|
$$

for all $t \geq 0$ (the value of $e(t)$ is not changing at instants of commutation $t_{i}$ ), consequently, the system is also stable.

Thus, in the ideal case $(D=V=0)$ the observer (4) provides global fixed-time stability of the estimation error for the system (2) with Lipschitz nonlinearity. If $D \neq 0$, then the rate of convergence is preserved in the system, but the overshoots during transients may have a complex behavior.

Remark 3. As we can conclude from Theorem 5.3, the lengths of intervals between switching $T_{i}$ are monotonously decreasing with $i \rightarrow+\infty$ and approaching zero in a finite time, then the dynamics exhibits a Zeno behavior, like in sliding mode control systems Boiko and Fridman (2005); Edwards and Spurgeon (1998); Filippov (1988); Fridman (2011); Levant (2010). In practice the switching with a frequency higher than the sampling frequency of the system (computer) is not possible, and the number of switches is always finite (the switching stops when $T_{i}$ becomes too small), thus the Zeno behavior in applications is never presented for (4).

Remark 4. The convergence speed can be increased by considering

$$
\mu_{i+1}=q^{\alpha} \mu_{i}
$$

with $\mu_{0}>1$ instead of (9), where $\mu_{0}=1$ always. The proofs stay almost the same in this case.

\subsection{Supervision algorithm for the noisy case}

Now let us consider the general case with $V \neq 0$. 


\subsubsection{Time-dependent switching}

From Corollary 5.2 we conclude that for any fixed $\mu_{i} \geq 1$ the estimation error in (2), (4) converges asymptotically to the ball of radius $r\left(\mu_{i}\right)=\sqrt{\frac{1}{\kappa \lambda_{\min }(P)}}\left(\rho_{d} \mu_{i}^{-1} D+\mu_{i}^{n-1} \rho_{v} V\right)$. Since $D>0$ and $V>0$ are external restrictions, and $P, \kappa, \rho_{d}, \rho_{v}$ are fixed under Assumption 4.1, then imposing a restriction that

$$
\rho_{d} D>(n-1) \rho_{v} V
$$

we can calculate the optimal value $\mu_{\min }$ of $\mu_{i}$, which minimize $r\left(\mu_{i}\right)$ (it is the solution of the equation $r^{\prime}\left(\mu_{\min }\right)=0$, and since direct computation shows that $r^{\prime \prime}\left(\mu_{\min }\right)>0$, then $\mu_{i}=\mu_{\min }$ is the minimum of $\left.r\left(\mu_{i}\right)\right)$ :

$$
\mu_{\min }=\sqrt[n]{\frac{1}{n-1} \frac{\rho_{d} D}{\rho_{v} V}},
$$

which is also related with the optimized gain obtained in Vasiljevic and Khalil (2008). Under (11) $\mu_{\min }>1$, and (11) can be guaranteed while solving LMIs for Assumption 4.1 by a proper selection of $\rho_{d}$ and $\rho_{v}$. The maximal value $\mu_{\max }$ of $\mu_{i}$, which does not lead to deterioration of the asymptotic estimation precision in (2), (4), can be found as the solution higher than $\mu_{\min }$ of the following equation:

$$
r\left(\mu_{\max }\right)=r\left(\mu_{0}\right),
$$

such a solution always exists under (11). For example, for $n=2$ and $\mu_{0}=1$ we obtain:

$$
\mu_{\min }=\sqrt{\frac{\rho_{d} D}{\rho_{v} V}}, \mu_{\max }=\frac{\rho_{d} D}{\rho_{v} V} .
$$

Therefore, in the noisy case the maximal number of switching $i^{*}>0$ can be calculated, and the switching stops either when $\mu_{i^{*}+1} \geq \mu_{\max }$ or when $T_{i^{*}+1} \leq T_{\min }$, where $T_{\min }>0$ is the time constant related with the maximal admissible frequency of commutation in the system. Hence,

$$
i^{*}=\text { floor }\left(\min \left\{\alpha^{-1} \log _{q} \mu_{\max }, i^{\prime}\right\}\right),
$$

where the function floor $(\cdot)$ returns the biggest integer not higher than the argument, and $i^{\prime}$ is the solution of the following equation:

$$
T_{\min }=-\frac{2}{\kappa q^{i^{\prime} \alpha}} \ln \left(\sqrt{\frac{\lambda_{\min }(P)}{\lambda_{\max }(P)}} \frac{q^{i^{\prime} \alpha(1-n)}}{q}\right) .
$$

Remark 5. Note that if the condition $\mu_{i^{*}+1} \geq \mu_{\max }$ is realized (or $i \geq i^{*}$ ), then after some dwell time the value of $\mu_{i}$ can be reset back to $\mu_{0}$ or $\mu_{\min }$, since a similar asymptotic accuracy is guaranteed in this case. This idea has been proposed in Ahrens and Khalil (2009). 


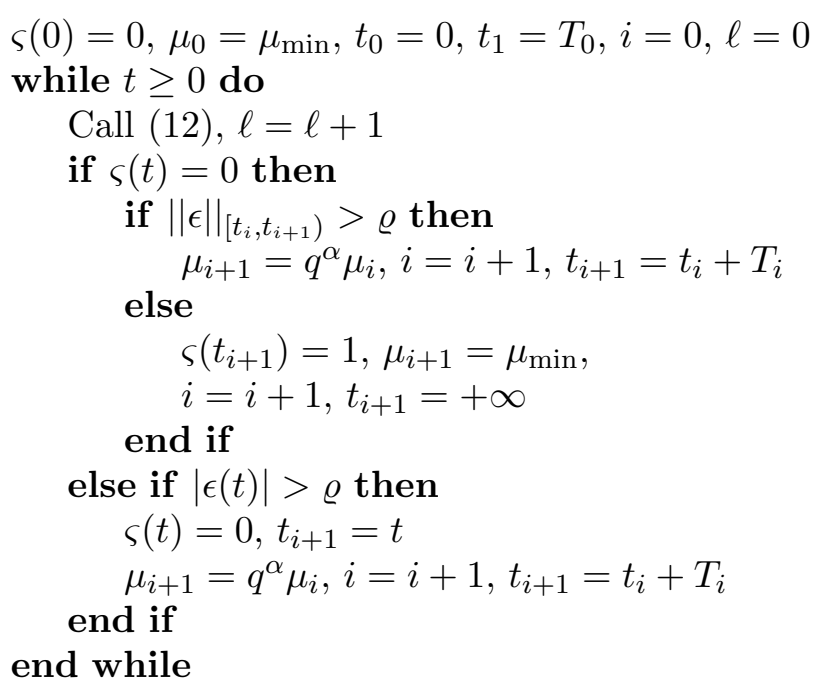

Algorithm 1: Output-based commutation

\subsubsection{Output-dependent switching}

Another possibility to orchestrate the observer gains is by analyzing the value of $\epsilon(t)=y(t)-c \hat{x}(t)=c^{T}[x(t)-\hat{x}(t)]+v(t)$, which is available for measurements, and which, according to Corollary 5.2, admits a monotonously decreasing upper bound for $i \in \mathbb{N}$. Indeed,

$$
\begin{gathered}
\left|c^{T}[x(t)-\hat{x}(t)]\right| \leq \sqrt{\frac{\lambda_{\max }(P)}{\lambda_{\min }(P)}} q^{-i}\left\|e\left(t_{0}\right)\right\|+\sqrt{\frac{1}{\kappa \lambda_{\min }(P)}} \rho_{v} V \\
\quad+\sqrt{\frac{1}{\kappa \lambda_{\min }(P)}}\left(\sqrt{\frac{\lambda_{\max }(P)}{\lambda_{\min }(P)}} \frac{q}{q-1}+\frac{1}{q^{i \alpha n}}\right) \rho_{d} D
\end{gathered}
$$

then for any $k>0$ there exists a finite time $\tau_{k}>0$ such that

$$
\begin{gathered}
|\epsilon(t)| \leq \varrho_{k} \quad \forall t \geq \tau_{k}, \\
\varrho_{k}=\sqrt{\frac{\lambda_{\max }(P)}{\lambda_{\min }(P)}} k+\left(1+\sqrt{\frac{1}{\kappa \lambda_{\min }(P)}} \rho_{v}\right) V \\
+\sqrt{\frac{1}{\kappa \lambda_{\min }(P)}}\left(\sqrt{\frac{\lambda_{\max }(P)}{\lambda_{\min }(P)}} \frac{q}{q-1}+k\right) \rho_{d} D .
\end{gathered}
$$

Therefore, a threshold $\varrho>0$ can be used to stop (8), (9) when acceleration phase is finished, next reset the value of $\mu_{i}$ to optimize the asymptotic precision (Remark 5), and finally to activate acceleration again if the signal has been changed and acceleration phase is needed again. The structure of this procedure is given in Algorithm 1, where the variable $\varsigma$ is used to memorize activation and deactivation of (8), (9). Note that the obtain algorithm is a kind of gain scheduling Rugh (1991). 


\subsection{Numerical implementation of (4)}

The main issue with application of the proposed observer with the switched gains is that $\mu_{i}$ is monotonously increasing in accordance with (9), and if the explicit Euler discretization method is used for implementation of (4), then such a realization may become unstable for some sufficiently high values of $\mu_{i}$. In order to avoid such a drawback, for the case $g(\cdot)=0$, the implicit Euler method is proposed to use in implementation of (4) (see Efimov, Polyakov, Levant, and Perruquetti (2016) for a discussion on advantages of the implicit Euler method other the explicit one for calculation of solutions of fixed-time stable systems, importance of application of the implicit Euler schemes for sliding-mode control realization is discussed in Acary, Brogliato, and Orlov (2012); Drakunov and Utkin (1989)). Let $h>0$ be the discretization step and $\hat{x}_{\ell}=\hat{x}(h \ell)$ be the value of estimate of the state at discrete time instant $h \ell$ with $\ell \in \mathbb{N}$, then in accordance with the implicit Euler method Butcher (2008):

$$
\hat{x}_{\ell+1}=O_{\mu_{i}}^{-1}\left\{\hat{x}_{\ell}+h\left[\varphi(y(h \ell), u(h \ell))+\mu_{i} M_{i}^{-1} l y(h \ell)\right]\right\},
$$

where $O_{\mu_{i}}=I_{n}-h A+\mu_{i} h M_{i}^{-1} l c^{T}$, and while $1+h \mu_{i} c^{T} S M_{i}^{-1} l \neq 0$, where $S=$ $\left(I_{n}-h A\right)^{-1}$, the inverse of $O_{\mu_{i}}$ can be derived using Sherman-Morrison formula:

$$
O_{\mu_{i}}^{-1}=S-\frac{S M_{i}^{-1} l c^{T} S}{h^{-1} \mu_{i}^{-1}+c^{T} S M_{i}^{-1} l} .
$$

Thus, the matrix $S$ can be calculated in advance, and the inverse of diagonal matrix $M_{i}$ is not costly. It is well known fact that the implicit Euler method for linear systems is converging for any $h$ Butcher (2008); Efimov, Polyakov, et al. (2016).

\section{Numerical comparison}

In order to illustrate the advantages of the proposed switched-gain observer (4), (8), (9) let us consider the problem of differentiation of a harmonic signal, and compare the solution obtained with (4) for a fixed $\mu_{i}$ and the well-known super-twisting differentiator Levant (2005):

$$
\begin{gathered}
\dot{\zeta}_{1}(t)=\zeta_{2}(t)-1.5 D^{0.5}\left|\zeta_{1}(t)-y(t)\right|^{0.5} \operatorname{sign}\left(\zeta_{1}(t)-y(t)\right), \\
\dot{\zeta}_{2}(t)=-1.1 D \operatorname{sign}\left(\zeta_{1}(t)-y(t)\right), \\
y(t)=f(t)+v(t),
\end{gathered}
$$

where $\zeta=\left[\zeta_{1} \zeta_{2}\right]^{T} \in \mathbb{R}^{2}$ is the state of differentiator, $f \in \mathbb{R}$ is the useful signal to be differentiated $\left(f=c^{T} x\right.$ for a suitably defined state $x$ in $\left.(2)\right), v$ is the measurement noise as previously, $\zeta_{2}$ is the estimate of $\dot{f}$, and $D$ is the Lipschitz constant of $\dot{f}$. For simulation purposes we will use:

$$
\begin{aligned}
& f(t)=20 t+\cos (t)+\sin (\pi t)+\left\{\begin{array}{ll}
2 & \text { if } t \in[0,3) \\
1002 & \text { if } t \geq 3
\end{array},\right. \\
& v(t)=V \sin (\omega t),
\end{aligned}
$$


where at the time instant $t=3$ an abrupt change in the signal is introduced in order to demonstrate the advantages of fixed-time convergence, then $D=1+\pi^{2}$ (this estimate is valid for $t \neq 3$ ), and take $V=0.1$. For (4) in this case

$$
A=\left[\begin{array}{ll}
0 & 1 \\
0 & 0
\end{array}\right], \varphi(\cdot)=g(\cdot)=0,
$$

and in order to calculate $l$ the values $\rho_{d}=\rho_{v}=1$ and $\kappa=1$ were selected $(\gamma=0)$, then the LMIs from Assumption 4.1 (Remark 1) are satisfied for

$$
l=\left[\begin{array}{ll}
2.615 & 2.103
\end{array}\right]^{T}, P=\left[\begin{array}{cc}
0.768 & -0.48 \\
-0.48 & 0.6
\end{array}\right] .
$$

Take $q=2, \alpha=1$ and the output-based supervision rule underlined in Algorithm 1 with $\varrho=0.5$, then $\mu_{\min }=10.42$ and $\mu_{\max }=108.69$. For the selected values of parameters the uniform global convergence time estimate $\bar{T}_{0}=9.11$. The super-twisting algorithm is implemented using the explicit Euler method Levant (2005) (the implicit realization is also possible as in Acary et al. (2012)), while for (4) the implicit Euler method based computation (12) is used. We also compare the results with the observer (4) with a constant value of $\mu_{i}=30$ (without the supervision algorithm (8), (9) and using the implicit Euler method for calculation of estimates).

For $h=0.01$ and $\omega=50$, the signal $f^{\prime}(t)$ in logarithmic scale and the corresponding estimates are shown in Fig. 1 together with behavior of $\mu_{i}$, the same results for $h=$ 0.001 and $\omega=100$ are presented in Fig. 2. The initial conditions for all observers have been selected to be zero. The value of $\mu_{i}$ is eventually increased by Algorithm 1 when a jump in the signal is detected at $t=3$. As we can conclude from these results, gain switching reduces peaking phenomenon and asymptotic noise sensitivity comparing with the constant gain case. The switched-gain observer also demonstrates by construction the fastest rate of convergence for big initial errors of estimation (it is not shown in simulation due to its clarity).

In order to quantify the comparison, the following performance indexes have been introduced:

$$
\begin{aligned}
J_{2,100 \%} & =\sqrt{\sum_{i=0}^{N}\left|f^{\prime}\left(t_{i}\right)-\hat{f}^{\prime}\left(t_{i}\right)\right|^{2}}, \\
J_{2,10 \%} & =\sqrt{\sum_{i=0.9 N}^{N}\left|f^{\prime}\left(t_{i}\right)-\hat{f}^{\prime}\left(t_{i}\right)\right|^{2}}, \\
J_{\infty} & =\max _{0 \leq i \leq N}\left|f^{\prime}\left(t_{i}\right)-\hat{f}^{\prime}\left(t_{i}\right)\right|,
\end{aligned}
$$

where $f^{\prime}\left(t_{i}\right)$ and $\hat{f}^{\prime}\left(t_{i}\right)$ are the derivative and its estimate provided by a differentiation algorithm at time instant $t_{i}=i h ; i=0,1, \ldots, N$ with $N$ being the number of steps used for simulation (the final time $t_{f}=N h=6 \mathrm{sec}$ ). Then $J_{2,100 \%}$ corresponds to a usual $L_{2}$ norm calculated for the error of differentiation during all time of simulation, the index $J_{2,10 \%}$ is calculated on the last $10 \%$ of simulation time interval in order to evaluate the asymptotic precision, and $J_{\infty}$ corresponds to $L_{\infty}$ norm of the total differentiation error quantifying the overshooting. The values of these performance indexes calculated in simulations are given in tables 1 and 2 . As we can conclude from 

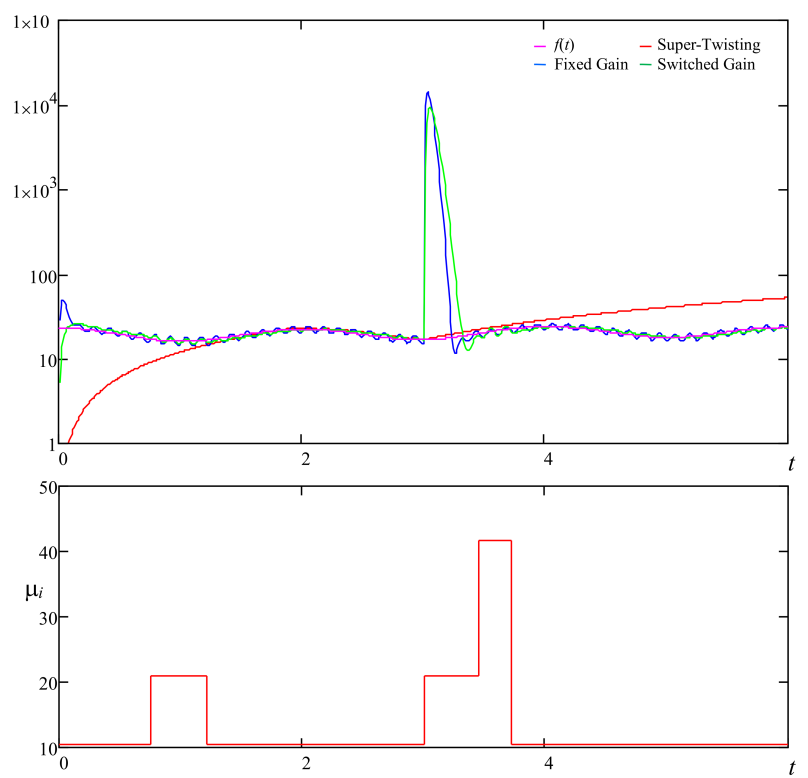

Figure 1. Results of simulation for $h=0.01$ and $\omega=50$
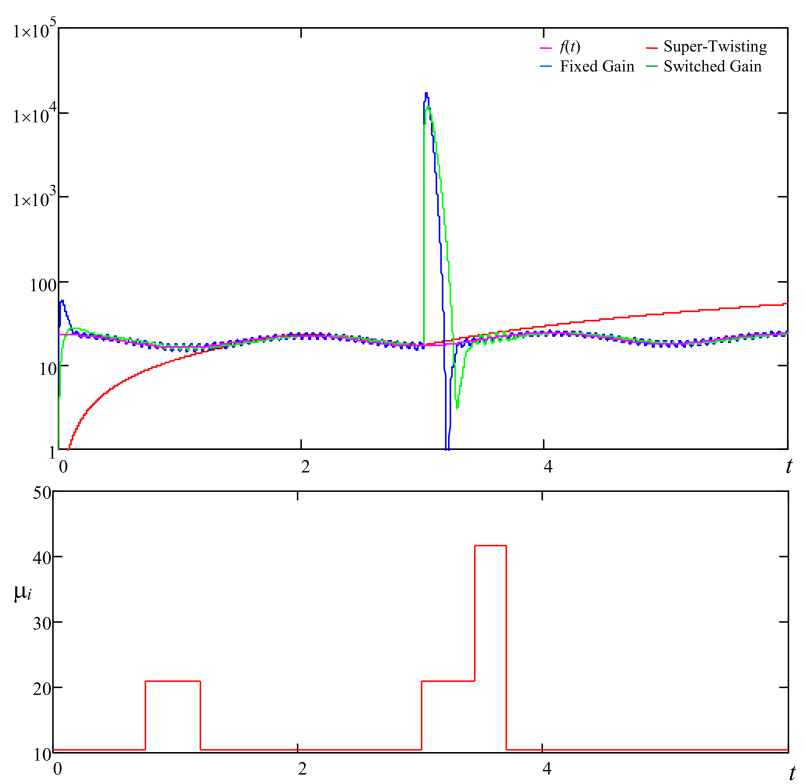

Figure 2. Results of simulation for $h=0.001$ and $\omega=100$ 


\begin{tabular}{|c|c|c|c|}
\hline & $J_{2,100 \%}$ & $J_{2,10 \%}$ & $J_{\infty}$ \\
\hline \hline Super-twisting & 336.5 & 210 & 29.2 \\
\hline Constant gains & 31684 & 10.6 & 14227 \\
\hline Switched gains & 23995 & 7.9 & 9175 \\
\hline
\end{tabular}

Table 1. Values of performance indexes for $h=0.01$ and $\omega=50$

\begin{tabular}{|c|c|c|c|}
\hline & $J_{2,100 \%}$ & $J_{2,10 \%}$ & $J_{\infty}$ \\
\hline \hline Super-twisting & 1071 & 665 & 29.4 \\
\hline Constant gains & 108555 & 29 & 16751 \\
\hline Switched gains & 89605 & 21.9 & 11563 \\
\hline
\end{tabular}

Table 2. Values of performance indexes for $h=0.001$ and $\omega=100$

these results, the super-twisting algorithm has good values of indexes $J_{2,100 \%}$ and $J_{\infty}$, but at the price that it is slowly converging, as we can clearly see from figures 1, 2 and the index $J_{2,10 \%}$. The observer with switched gains has better performance than with the constant ones (in terms of precision and peaking).

\section{Conclusion}

The problem of acceleration of the estimation convergence rate for a class of nonlinear systems in the output canonical form by switching among different values of observer gains was investigated. The presence of bounded matched disturbances, Lipschitz uncertainties and measurement noises were taken into account. The proposed switched-gain observer ensures global fixed-time stability of the estimation error at the origin in the noise-free case. In the presence of noise two modified commutation strategies for the observer gains are proposed, first one ensures peaking avoiding for the initial phase, convergence acceleration and optimization of asymptotic precision of estimation, second strategy is output signal based and serves for the same purposes. The results are illustrated by computer simulation and comparison in planar case with super-twisting differentiator and a linear high-gain observer with constant gains.

\section{References}

Acary, V., Brogliato, B., \& Orlov, Y. V. (2012). Chattering-free digital sliding-mode control with state observer and disturbance rejection. IEEE Transactions on Automatic Control, $57(5), 1087-1101$.

Ahrens, J. H., \& Khalil, H. K. (2009). High-gain observers in the presence of measurement noise: A switched-gain approach. Automatica, 45(4), 936-943.

Ananyevskii, I. (2001). Limited control of a rheonomous mechanical system under uncertainty. Journal of Applied Mathematics and Mechanics, 65(5), 785-796.

Ananyevskii, I. (2003). Control synthesis for linear systems by methods of stability theory of motion. Difierential Equations, 39(1), 1-10.

Andrieu, V., Praly, L., \& Astolfi, A. (2009). High gain observers with updated gain and homogeneous correction terms. Automatica, 45(2), 422-428.

Boiko, I., \& Fridman, L. (2005). Analysis of chattering in continuous sliding-mode controllers. IEEE Transactions on Automatic Control, 50(9), 1442-1446.

Boizot, J. N., Busvelle, E., \& Gauthier, J.-P. (2010). An adaptive high-gain observer for nonlinear systems. Automatica, 46(9), 1483-1488. 
Butcher, J. C. (2008). Numerical methods for ordinary differential equations (2nd ed.). New York: John Wiley \& Sons.

Crassidis, J. L., \& Junkins, J. L. (2012). Optimal estimation of dynamic systems (2nd ed.). CRC Press.

Cruz-Zavala, E., Moreno, J., \& Fridman, L. (2011). Uniform robust exact differentiator. IEEE Transactions on Automatic Control, 56(11), 2727-2733.

Drakunov, S., \& Utkin, V. (1989). On discrete-time sliding modes. IFAC Proceedings Volumes, 22(3), 273-278. (Nonlinear Control Systems Design, Capri, Italy, 14-16 June 1989)

Dvir, Y., \& Levant, A. (2015a, Oct). Accelerated twisting algorithm. Automatic Control, IEEE Transactions on, 60(10), 2803-2807.

Dvir, Y., \& Levant, A. (2015b). Sliding mode order and accuracy in sliding mode adaptation and convergence acceleration. In X. Yu \& M. Önder Efe (Eds.), Recent advances in sliding modes: From control to intelligent mechatronics (Vol. 24, pp. 129-153). Springer International Publishing.

Edwards, C., \& Spurgeon, S. (1998). Sliding mode control: theory and applications. Taylor and Francis.

Efimov, D., Levant, A., Polyakov, A., \& Perruquetti, W. (2016). On acceleration of asymptotically stable homogeneous systems. In Proc. 55th ieee conference on decision and control $(c d c)$. Las Vegas.

Efimov, D., Levant, A., Polyakov, A., \& Perruquetti, W. (2017). Supervisory acceleration of convergence for homogeneous systems. International Journal of Control, 0(0), 1-11.

Efimov, D., Polyakov, A., Levant, A., \& Perruquetti, W. (2016). Discretization of asymptotically stable homogeneous systems by explicit and implicit Euler methods. In Proc. 55th ieee conference on decision and control (cdc). Las Vegas.

Elbeheiry, E. M., \& Elmaraghy, H. A. (2003). Robotic manipulators state observation via one-time gain switching. Journal of Intelligent and Robotic Systems, 38, 313-344.

Filippov, A. (1988). Differential equations with discontinuous righthand sides. Kluwer Academic Publishers.

Fridman, L. (2011). Sliding mode enforcement after 1990: Main results and some open problems. In R. I. L. Fridman J. Moreno (Ed.), (Vol. 412, pp. 3-57). Berlin Heidelberg: Springer - Verlag.

Goebel, R., Sanfelice, R., \& Teel, A. (2009). Hybrid dynamical systems. IEEE Control Systems Magazine, 29(2), 28-93.

Khalil, H. K. (1996). Nonlinear systems. Upper Saddle River: Prentice-Hall.

Levant, A. (2005). Homogeneity approach to high-order sliding mode design. Automatica, $41(5), 823-830$.

Levant, A. (2010). Chattering analysis. IEEE Transactions on Automatic Control, 55(6), 1380-1389.

Lin, Y., Sontag, E. D., \& Wang, Y. (1996). A smooth converse lyapunov theorem for robust stability. SIAM Journal on Control and Optimization, 34(1), 124-160.

Lopez-Ramirez, F., Efimov, D., Polyakov, A., \& Perruquetti, W. (2016). Fixed-time output stabilization of a chain of integrators. In Proc. 55th ieee conference on decision and control (cdc). Las Vegas.

Luenberger, D. G. (1979). Introduction to dynamic systems: theory, models, and applications. Wiley.

Moreno, J., \& Osorio, A. (2012). Strict lyapunov functions for the super-twisting algorithm. IEEE Transactions on Automatic Control, 57(4), 1035-1040.

Moulay, E., \& Perruquetti, W. (2006). Finite time stability and stabilization of a class of continuous systems. Journal of Mathematical Analysis and Application, 323(2), 1430-1443.

Moulay, E., \& Perruquetti, W. (2008). Finite time stability conditions for non autonomous continuous systems. International Journal of Control, 81(5), 797-803.

Polyakov, A. (2012). Nonlinear feedback design for fixed-time stabilization of linear control systems. IEEE Transactions on Automatic Control, 57(8), 2106-2110.

Poznyak, A., Fridman, L., \& Bejarano, F. J. (2004). Mini-max integral sliding-mode control 
for multimodel linear uncertain systems. IEEE Transactions on Automatic Control, $49(1)$, $97-102$.

Ríos, H., \& Teel, A. (2016). A hybrid observer for uniform finite-time state estimation of linear systems. In In proc. 55th ieee conference on decision and control (cdc). Las Vegas.

Roxin, E. (1966). On finite stability in control systems. Rendiconti del Circolo Matematico di Palermo, 15, 273-283.

Rugh, W. J. (1991). Analytical framework for gain scheduling. IEEE Control Systems, 11(1), 79-84.

Sanfelice, R. G., \& Praly, L. (2011). On the performance of high-gain observers with gain adaptation under measurement noise. Automatica, 47(10), 2165-2176.

Sontag, E. (1998). Mathematical control theory: Deterministic finite dimensional systems (2nd ed.). Springer.

Utkin, V. I. (1992). Sliding modes in control optimization. Berlin: Springer-Verlag.

Vasiljevic, L. K., \& Khalil, H. K. (2008). Error bounds in differentiation of noisy signals by high-gain observers. Systems \& Control Letters, 57(10), 856-862. 